\title{
Electrophysiological investigations of cephalic tetanus
}

\author{
RICARDO GARCIA-MULLIN AND ROBERT B. DAROFF \\ From the Department of Neurology, University of Miami School of Medicine, \\ Jackson Memorial Hospital, and the Neurology Service, Veterans Administration Hospital, \\ Miami, Florida, U.S.A.
}

SUMMARY Electrophysiological studies of a patient with cephalic tetanus, manifested by trismus and unilateral facial palsy, revealed that the trismus resulted from strictly unilateral masseter spasm which stopped during sleep, a normal silent period in the involved masseter muscles, initially prolonged distal latency of the paretic orbicularis muscles, and abnormal continuous muscle activity in the paretic facial muscles which stopped during sleep and after facial nerve block. We conclude that the facial paralysis was probably secondary to peripheral nerve dysfunction and the continuous muscle potentials represented enhanced activity of the nucleus. An intensity spectrum of the inhibitory defect in tetanus is postulated.

Cephalic tetanus is defined as a form of the disease in which trismus is accompanied by paralytic involvement of one or more cranial nerves (Bagratuni, 1952). The tetanus may be generalized or local, with trismus in the latter constituting the sole manifestation of spasm. The causative injury is usually on the head or face and the ipsilateral facial nerve is most commonly affected (Park, 1970). The neurophysiological mechanism underlying the cranial nerve palsies has been an enigmatic feature in a disorder otherwise characterized by spasms secondary to abnormally enhanced motoneuronal activity (Brooks, Curtis, and Eccles, 1957). We are? herein reporting a patient with cephalic tetanus who demonstrated a unique type of constant
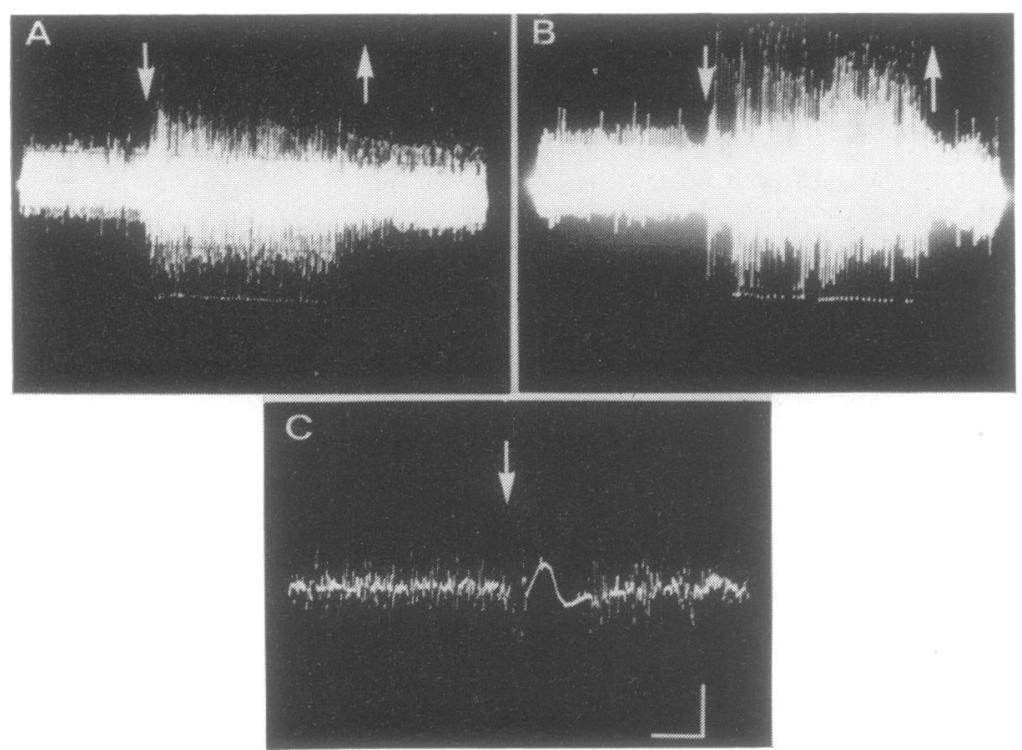

FIG. 1 EMG recording from left masseter muscle demonstrating abnormal continuous activity. In $\mathrm{A}$ and $\mathrm{B}$, the arrows indicate beginning and end of opening mouth and biting, respectively; both resulted in an increase in the frequency and amplitude of the muscle action potentials. In $\mathrm{C}$, the jaw jerk (arrow) was followed by a normal silent period. (Calibration: $200 \mu \mathrm{V}$ and $1 \mathrm{sec}$ in $\mathrm{A}$ and B; $200 \mu \mathrm{V}$ and $50 \mathrm{msec}$ for $\mathrm{C}$.) 
motor unit activity from partially paralysed facial muscles. Our electrodiagnostic studies in this patient provide information relevant to the pathophysiology of both the cranial nerve palsy and trismus in tetanus.

\section{CASE REPORT}

A 59 year old man fell and lacerated the left side of his face eight days before admission to Jackson Memorial Hospital. After the injury, he covered the wound with cloth and did not seek medical attention. Purulent drainage commenced four days later and during the evening before admission he noted tightness of the jaw. On the morning of admission he was unable to open his mouth and facial asymmetry developed. He never had a complete course of tetanus immunization but on two separate occasions after extremity lacerations, five and two years previously, he received $0.5 \mathrm{ml}$. tetanus toxoid.

Examination on admission revealed an alert, oriented, cooperative patient with normal vital signs. He had an infected wound lateral to the left orbit which extended from the level of the canthus $3 \mathrm{in}$. onto the forehead. There was no local inflammatory process in the pterygoid fossa or temporomandibular joint areas. All muscles innervated by the left facial nerve were partially paralysed resulting in distinct asymmetry of the face. Voluntary effort produced a trace contraction of the left frontalis and orbicularis oculi muscles and a very weak contraction of the orbicularis oris, but none of the zygomaticus, risorius, or platysma muscles. There were no clinically detectable abnormal movements in any of

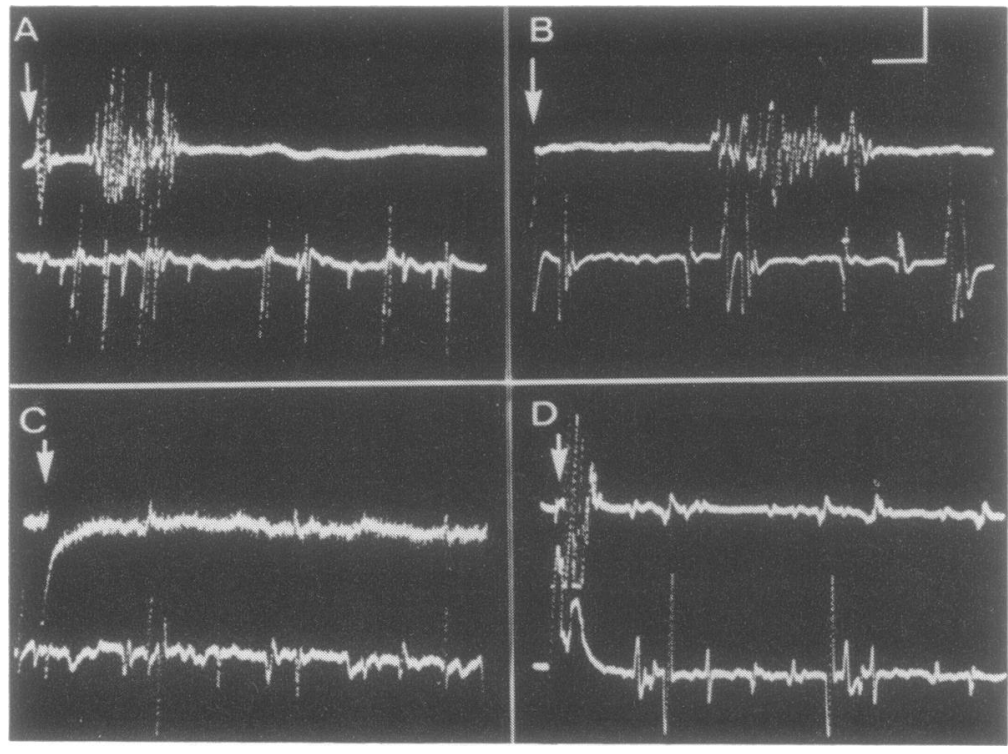

FIG. 2 The top EMG

tracings in $\mathrm{A}$ and $\mathrm{B}$ are from the right orbicularis oculi and the bottom tracings from the left masseter muscle. Stimulation of the right supraorbital nerve in A (arrow) was followed by normal early and late reflex responses in the right orbicularis oculi muscle. In $\mathrm{B}$, stimulation of the left supraorbital nerve (arrow) was followed by normal late reflex response in right orbicularis oculi muscle. (Calibration: $100 \mu \mathrm{V}$ and 20 msec for $\mathrm{A}$; $100 \mu \mathrm{V}$ and $10 \mathrm{msec}$ for $\mathrm{B}$.) In $\mathrm{C}$, the top tracing is from the left orbicularis oculi and bottom from the left masseter muscle. Stimulation of left supraorbital nerve (arrow) produced no reflex response in either muscle. In $\mathrm{D}$, top tracing is from left orbicularis oris and bottom from left masseter muscle. Stimulation of left facial nerve (arrow) produced a direct motor response in the orbicularis oris which was not followed by a silent period. The deflection noted in the masseter represents direct muscle stimulation. (Calibration: $20 \mu \mathrm{V}$ and $10 \mathrm{msec}$ for $\mathrm{C} ; 100 \mu \mathrm{V}$ and $20 \mathrm{msec}$ for $\mathrm{D}$.) 
the left facial muscles. Trismus was marked and the mouth could not be opened more than a $5 \mathrm{~mm}$ distance between upper and lower teeth. The remainder of the cranial nerve examination revealed no abnormalities.

He had normal muscular tone and strength in all four extremities, with symmetrical tendon jerks and flexor plantar responses. We noted no spontaneous or stimulus-induced extremity muscle spasms and found no abnormalities on sensory testing. Complete blood count, urinalysis, serum electrolytes, total serum protein, calcium, phosphorus, cholesterol, glucose, blood urea nitrogen, serum uric acid, creatinine, bilirubin, LDH, and SGOT were normal. Radiographs of the chest and skull were normal.

Surgical consultants debrided and packed the wound, which was sutured 15 days later with good healing. The patient received 2,750 units of human antitetanus globulin and $1 \mathrm{ml}$. tetanus toxoid intramuscularly on admission, a second injection of $0.2 \mathrm{ml}$. toxoid on discharge three weeks later, and another $0.5 \mathrm{ml}$. four weeks after discharge. We instituted oral diazepam (10 mg every six hours) and intramuscular procaine penicillin $(1 \cdot 3$ million units every six hours) on admission and continued these drugs for 17 days.

The trismus and facial paralysis improved and upon discharge 22 days later the distance between the teeth upon opening the mouth was $12 \mathrm{~mm}$. Taste, which could not be examined previously because of trismus, was now normal on both sides. The trismus completely disappeared eight weeks after admission but the left facial paresis was present, although minimally, at 10 weeks.

ELECTROPHYSIOLOGICAL STUDIES We used bipolar needle electrodes (Disa 9013K0801 and 9013K0802), a Disa 3 channel electromyograph, and a Tektronix 565 oscilloscope for electromyography (EMG). For stimulation of the facial and supraorbital nerves we used a disc surface electrode $9 \mathrm{~mm}$ in diameter taped
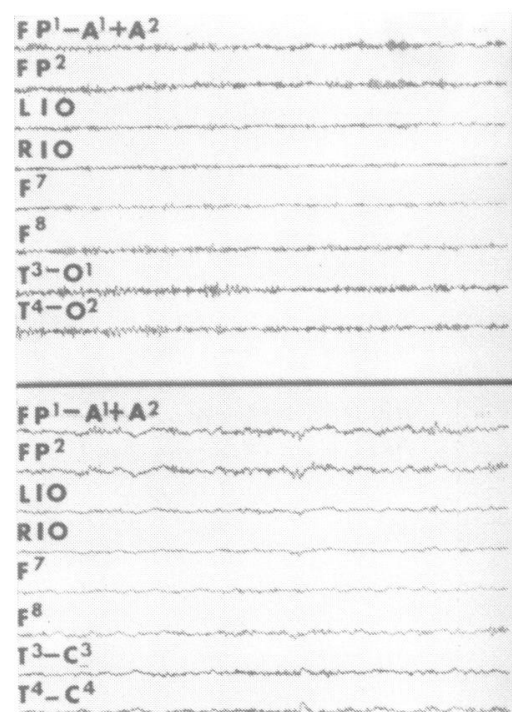

\begin{tabular}{l}
\hline$P^{1}-A^{1}+A^{2}$ \\
$F P^{2}$ \\
$L 10$ \\
$R 10$ \\
$F^{7}$ \\
$F^{8}$ \\
$\frac{r^{3}-C^{3}}{r^{4}-C^{4}}$ \\
\hline
\end{tabular}
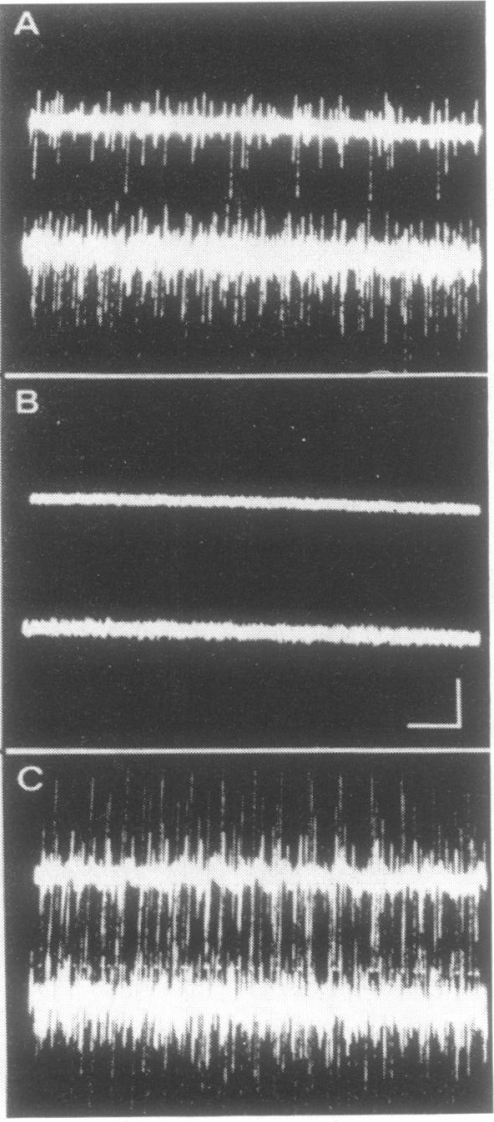

FIG. 3 Simultaneous EEG and $E M G$ recordings from left masseter (top) and left orbicularis oris muscles (bottom). There was abnormal continuous activity in both muscles at rest while subject was awake in $\mathrm{A}$, and after arousal in $\mathrm{C}$. There was complete cessation of all muscle activity during light sleep in $\mathrm{B}$. (Calibration: $50 \mu \mathrm{V}$ and $1 \mathrm{sec}$ for the EEG; $50 \mu V$ and 100 msec for the EMG.) 
to the skin with a silver plate $4 \mathrm{~cm} \times 3 \mathrm{~cm}$ over the sternum as the indifferent electrode. Square pulses of 0.1 to 0.5 msec duration and voltages from 50 to $500 \mathrm{~V}$ were delivered with a Disa Multistim stimulator.

1. There was abnormal EMG activity in the left masseter muscle at rest which consisted of bi- or triphasic muscle action potentials with amplitudes ranging from 50 to $800 \mu \mathrm{V}$, and durations from 3 to $6 \mathrm{msec}$, firing at frequencies between 12 and 20 per second. There was no tendency for this activity to become grouped in doublets or multiplets, and it persisted unchanged in amplitude and frequency during continuous recordings for periods of up to 30 minutes. Voluntary biting as well as effort to open the mouth produced an increase in the frequency and amplitude of the muscle action potentials (Fig. 1, A and B). Tapping of the jaw elicited a reflex jerk with a synchronous increase in the number of muscle action potentials which was followed by a silent period lasting $75 \mathrm{msec}$ (Fig. 1C). Stimulation of the supraorbital and/or facial nerves on either side failed to change the pattern of muscle action potential activity in this left masseter muscle (Fig. 2). The activity disappeared completely during sleep (Fig. 3).

In follow-up studies performed two and six weeks later, the EMG activity of the left masseter persisted, although decreasing in the number, amplitude, and frequency of units. Fifteen days after the initial examination only one or two motor units fired at frequencies of 12 to 16 per second with amplitudes around $250 \mu \mathrm{V}$; there were transient periods of total silence lasting a few seconds. At 10 weeks after admission, no abnormal activity was recorded from this muscle.

2. The right masseter muscle was silent at rest, and biting produced a normal interference pattern. No activity was present during effort to open the mouth.

3. We recorded abnormal activity in the left orbicularis oris and orbicularis oculi muscles which consisted of biphasic and triphasic muscle action potentials with amplitudes from 100 to $250 \mu \mathrm{V}$, and durations between 2 and $4 \mathrm{msec}$, firing at frequencies from 30 to 40 per second (Fig. 4). This EMG activity persisted unchanged in amplitude or frequency during prolonged periods of observation up to one hour. Voluntary contraction increased the frequency and amplitude of the muscle action potentials but no polyphasic potentials of prolonged duration were found. No reflex response was noted in these muscles after left supraorbital nerve stimulation (Fig. 2C) and no silent period of this EMG activity followed stimulation of the left facial nerve (Fig. 2D). However, both light sleep (Fig. 3) and lidocaine infiltration of the cervicofacial division of the left facial nerve (Fig. completely abolished the spontaneous activity in the left orbicularis muscles. We found no decrement of increment in the activity of the left orbicularis ocu muscle recorded with surface electrodes when trains of supramaximal stimuli of $2 \mathrm{sec}$ duration and fre-

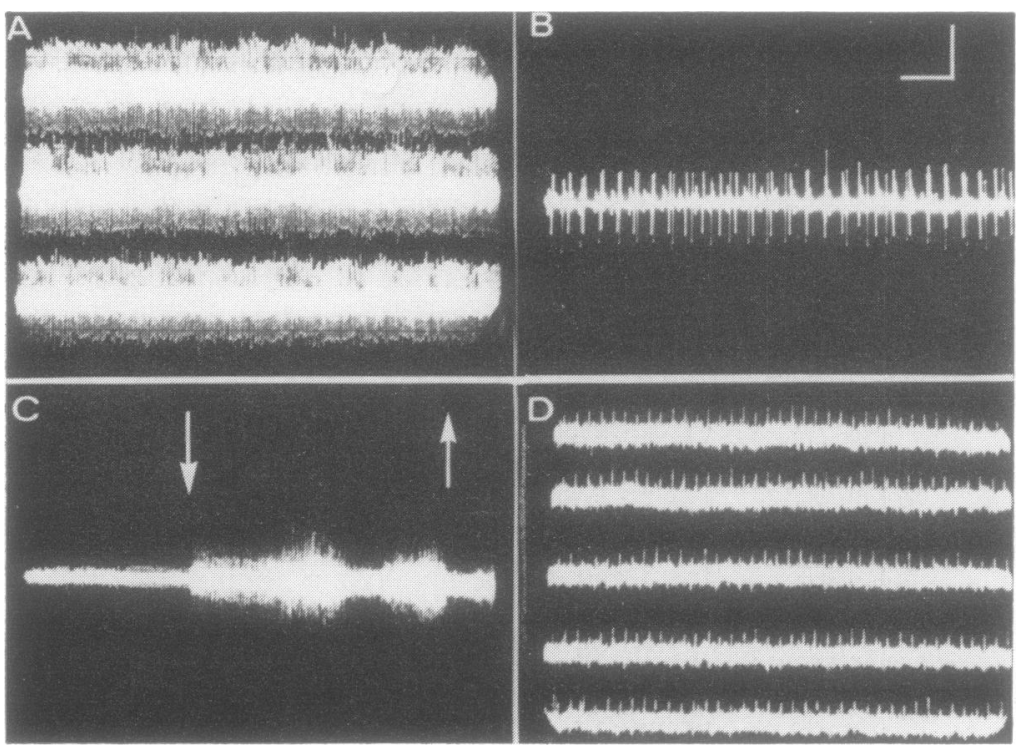

FIG. 4 A and B demonstrate the abnormal continuous EMG activity at different sweep speeds from the orbicularis oris muscle at rest. In $\mathrm{C}$, the muscle potentials increased in amplitude and frequency during attempts at voluntary contraction (whistling). (Calibration: $100 \mu V$ and 1 sec in $\mathrm{A}$; $100 \mu \mathrm{V}$ and $100 \mathrm{msec}$ in $\mathrm{B}$, $500 \mu \mathrm{V}$ and $1 \mathrm{sec}$ in $\mathrm{C}$.) In $\mathrm{D}$, the EMG recordings of the left orbicularis oculi muscle during five successive sweeps demonstrated the continuous muscle activity. The high frequency, persistence, and regularity of this activity did not resemble fibrillations. (Calibration: $20 \mu \mathrm{V}$ and $100 \mathrm{msec}$.) 
quencies of 3,10 , and 30 per second were delivered to the left facial nerve.

Follow-up studies six weeks after admission revealed that the frequency of the spontaneous activity in the left orbicularis muscles had decreased to levels between 25 and 30 per second.

Distal motor latency at the left orbicularis oculi muscle after stimulation of the left facial nerve at the angle of the jaw was $6 \mathrm{msec}$ on admission, $5 \mathrm{msec}$ at two weeks, and $3.2 \mathrm{msec}$ at six weeks.

4. We found no abnormal activity in the right orbicularis oris or oculi muscles. There were normal interference patterns on voluntary contraction;

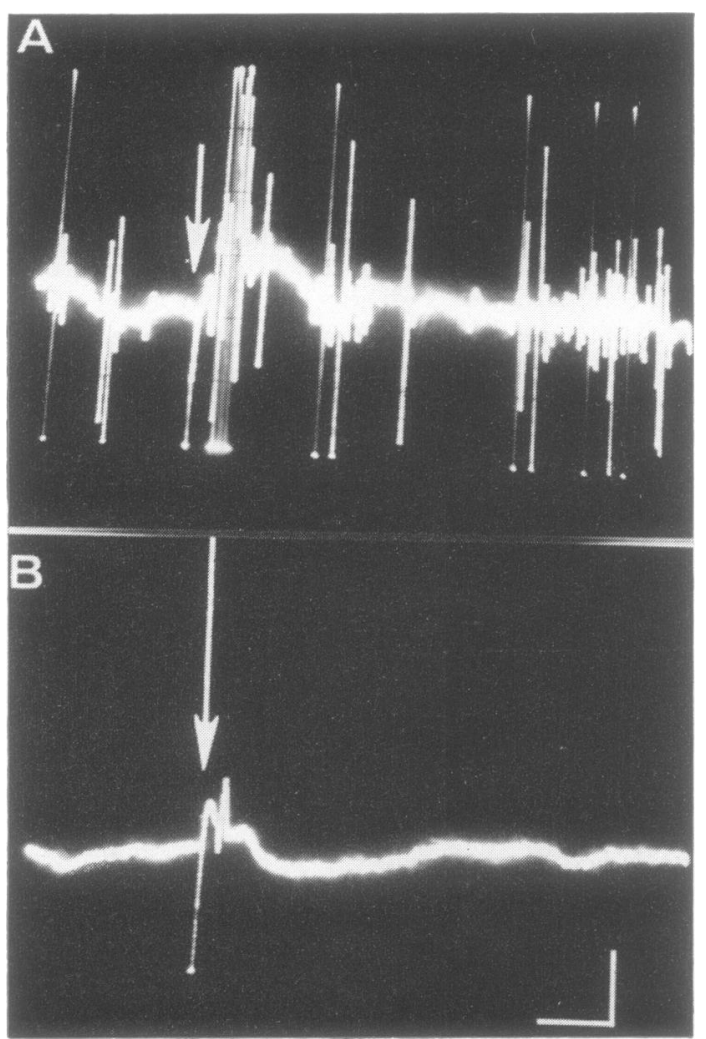

FIG. 5. EMG recording from left orbicularis oris muscle before (A) and after (B) infiltration of the cervicofacial division of the left facial nerve with $2 \mathrm{ml} .1 \%$ lidocaine. Arrow indicates supramaximal stimulation of the left facial nerve proximal to the area of block. In $\mathrm{B}$, there was complete disappearance of spontaneous activity and markedly reduced response to nerve stimulation after lidocaine block. (Calibration: $50 \mu \mathrm{V}$ and $20 \mathrm{msec}$.) normal early and late responses following stimulation of the right supraorbital nerve; and normal late responses following stimulation of the left supraorbital nerve (Fig. 2, A and B).

\section{DISCUSSION}

Tetanus toxin produces muscle spasms by blocking the postsynaptic inhibition of motoneurones by interneurones (Brooks, et al., 1957); in spinal interneurones the toxin reduces the amount of glycine released from inhibitory terminals (Curtis and de Groat, 1968). The clinical expressivity of the effects of tetanus toxin on the nervous system ranges from severe generalized involvement to milder forms of localized spasms (Struppler, Struppler, and Adams, 1963) such as were manifested in our patient. Our electrodiagnostic findings indicate a similar intensity spectrum of the inhibitory defect basic to the disorder. Struppler et al. (1963) reported the absence of a silent period in the masseter muscles after the jaw jerk as characteristic of tetanic trismus; however, our patient with trismus had a normal silent period. Similarly, whereas trismus and extremity muscle spasms have been observed clinically to persist during sleep, we demonstrated the total absence of masticatory and facial muscle activity during sleep. Thus, the inhibitory defect in at least local tetanus need not be as intense as that usually associated with generalized and severe forms of the disorder. We suspect that, if left untreated, the tetanus may have evolved into a generalized type with the usual electrophysiological evidence of profound loss of inhibition.

The abnormal muscle action potential activity recorded in the partially paralysed facial muscles of the patient has not, to our knowledge, been reported previously. He did not have the clinical manifestations of facial hemispasm which occasionally occurs in cephalic tetanus (Watkins, 1939), and the electromyographic pattern was inconsistent with hemispasm (Bratzlavsky and Vander Eecken, 1971). The continuous activity should also be differentiated from the facial myokymia found in cases of multiple sclerosis and brain-stem gliomas, where the firing pattern is more bizarre and complex with a tendency to grouping of muscle action potentials into multiplets (Matthews, 1966). 
The absence of a silent period in the continuously active left orbicularis oculi muscle after left facial nerve stimulation is not specific for tetanus but may occur in cases of facial myokymia (Garcia-Mullin, unpublished observations).

The cranial nerve palsies in cephalic tetanus could share the same mechanism as the motor paralysis of extremities that occasionally occurs in severe generalized tetanus. Kaeser and colleagues $(1968,1970)$ regarded the latter as secondary to a block in neuromuscular transmission possibly due to impaired release of acetylcholine. Watkins (1939) considered the facial paralysis of cephalic tetanus as a peripheral lesion on the basis of associated ipsilateral taste loss in his patient. Park (1970) and others, however, postulated nuclear dysfunction. We found no abnormal decrement or increment in the evoked facial muscle potentials after trains of different frequency stimuli delivered to the facial nerves. This mitigated against a causative defect in neuromuscular transmission. The prolonged distal latency of orbicularis contraction early in the course of the illness, which improved after treatment, probably indicates the site of paralysis to be along the peripheral course of the VII nerve (Gilliatt and Taylor, 1959; Taverner, 1965), but may also be compatible with dysfunction affecting predominantly the larger motoneurones of the VII nerve nucleus.

Lidocaine block of the facial nerve eliminated the abnormal continuous action potentials recorded in the paralysed orbicularis muscle innervated by that nerve. This indicated that the origin of the muscular activity was proximal to the block. The cessation of these potentials during sleep implicates a causative central or nuclear abnormality. We suggest that neurones within the left facial nucleus, presumably released from inhibitory control, fired continuously and resulted in the recorded abnormal resting activity in the muscles. We are unaware of electromyographic studies of the facial muscles in risus sardonicus, but suspect that this condition may represent an advanced manifestation of abnormal release of facial motoneurones. Perhaps the coexisting peripheral facial paralysis masked the clinical appearance of risus sardonicus in our patient.

Our study also demonstrated that clinically distinct tetanic trismus may result from strictly unilateral masticatory muscle spasm.

\section{REFERENCES}

Bagratuni, L. (1952). Cephalic tetanus with report of a case. British Medical Journal, 1, 461-463.

Bratzlavsky, M., and Eecken, H. Vander (1971). Hémispasme facial primitif: corrélations cliniques et électromyographiques. Acta Neurologica Belgica, 71, 365-382.

Brooks, V. B., Curtis, D. R., and Eccles, J. C. (1957). The action of tetanus toxin on the inhibition of motoneurones. Journal of Physiology, 135, 655-672.

Curtis, D. R., and de Groat, W. C. (1968). Tetanus toxin and spinal inhibition. Brain Research, 10, 208-212.

Gilliatt, R. W., and Taylor, J. C. (1959). Electrical changes following section of the facial nerve. Proceedings of the Royal Society of Medicine, 52, 1080-1083.

Kaeser, H. E., Müller, H. R., and Friedrich, B. (1968). The $\omega$ nature of tetraplegia in infectious tetanus. European Neurology, 1, 17-27.

Kaeser, H. E., and Saner, A. (1970). The effect of tetanus toxin on neuromuscular transmission. European Neurology, 3, 193-205.

Matthews, W. B. (1966). Facial myokymia. Journal of Neurology, Neurosurgery, and Psychiatry, 29, 35-39.

Park, D. M. (1970). Cranial nerve palsies in tetanus: cephalic tetanus. Journal of Neurology, Neurosurgery, and Psychiatry, 33, 212-215.

Struppler, A., Struppler, E., and Adams, R. D. (1963). Local tetanus in man. Its clinical and neurophysiological characteristics. Archives of Neurology, 8, 162-178.

Taverner, D. (1965). Electrodiagnosis in facial palsy. Archives of Otolaryngology, 81, 470-477.

Watkins, A. L. (1939). Facial paralysis in cephalic tetanus. Archives of Neurology and Psychiatry (Chicago), 41 788-792. 